\title{
CORTICAL ACTIVATION PATTERNS OF CUE-PACED FOOT MOVEMENT IN SUBACUTE STROKE PATIENTS
}

\author{
T. Solis-Escalante ${ }^{1}$, J. M. Belda-Lois ${ }^{2}$, and G. R. Müller-Putz ${ }^{1}$ \\ ${ }^{1}$ Institute for Knowledge Discovery, Graz University of Technology, Austria \\ ${ }^{2}$ Instuto de Biomecánica de Valencia, Universitat Politécnica de Valencia, Spain \\ teodoro.solisescalante@ tugraz.at
}

\begin{abstract}
Limb movement is associated with well defined cortical activation patterns. Structural and functional changes in the brain affect the characteristics of these patterns (strength and topography). Novel strategies for poststroke motor rehabilitation could monitor cortical activity as an additional index of engagement and/or recovery. In this work we analyze differences in cortical activation related to movements of the affected vs. unaffected foot (dorsiflexion). Our results show stronger cortical activation during movements of the affected foot, and stronger responses at the vertex. Online assessment of cortical activation and the experiment described in this work could be added to traditional motor rehabilitation.
\end{abstract}

Keywords: Event-related (de)synchronization, Postmovement beta rebound, Subacute stroke, EEG

\section{Introduction}

Novel strategies for improving post-stroke motor rehabilitation may monitor cortical activation during therapy $[1,2]$. Cortical activation patterns related to limb movement are visible in the EEG as changes in the amplitude of sensorimotor rhythms. These changes are described as eventrelated desyncrhonization (ERD) and event-related synchronization (ERS) [3]. In general, ERD can be interpreted as a correlate of an active cortical area, while ERS can be associated with a deactivated or inhibited state of large neuronal networks. After a stroke, structural and functional changes in the brain affect the characteristics of ERD and ERS patterns, i.e., strength and topography $[4,5,6]$. In this work, we investigate the cortical activation patterns of subacute stroke patients during foot movements of the affected and unaffected side of their body.

\section{Methods}

Experimental paradigm and data acquisition: Eleven subacute stroke patients (ischemic stroke, time after lesion $8 \pm 2$ months, age $63 \pm 14$ yr., right handed, 5 female, 8 with left hemiparesis) were recruited by the Instituto de Biomecánica de Valencia, Spain. The participants' task was to perform left or right foot dorsiflexions following cues shown on a computer screen (8 runs with 10 movements per leg). Movement side (left and right) and duration ( 3 to $4 \mathrm{~s}$ ) were randomized. Online feedback, representing muscle activity, was presented to assist patients with the execution of the foot movements. The experiment

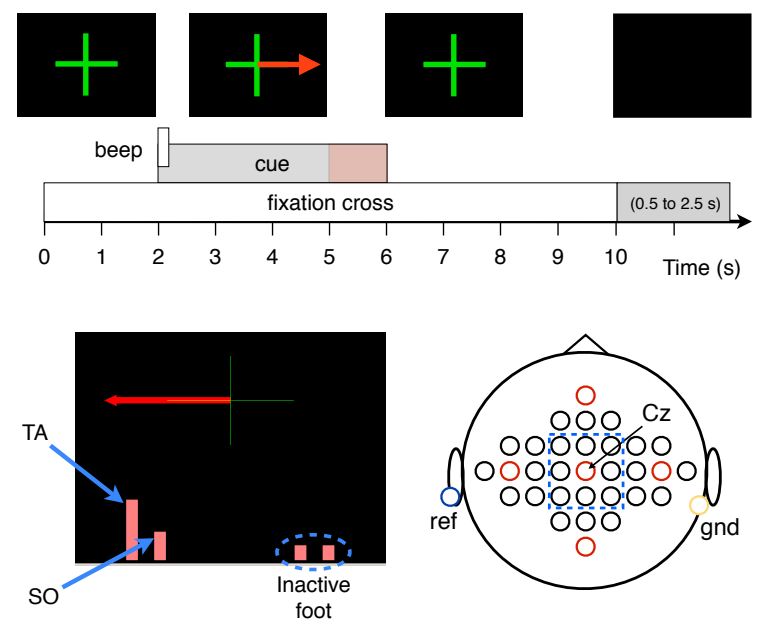

Figure 1: Experimental paradigm, online feedback, and electrode montage. Top: Schematic of the paradigm timing. Bottom left: Online feedback shows the muscle activity of both legs. Bottom right: Thirty-one electrodes around the vertex (electrode position $\mathrm{Cz}$ ) used to record EEG. Electrode positions in red are $\mathrm{Fz}, \mathrm{C} 3, \mathrm{Cz}, \mathrm{C} 4$, and $\mathrm{Pz}$.

was approved by the local ethics committee and all participants gave written informed consent. EEG was recorded from 31 sites using a custom cap (Easycap, Herrsching, Germany) and active electrodes (g.tec, Schiedelberg, Austria). The reference electrode was on the left earlobe and the ground electrode was on the right mastoid. EMG was recorded from each leg: extensor hallucis longus (HL), tibialis anterior (TA), soleus (SO), and gastrocnemius (GA). Two monopolar channels were recorded from each muscle using adhesive $\mathrm{Ag} / \mathrm{AgCl}$ electrodes (reference on the right hip). Signals were acquired at $1.2 \mathrm{kHz}$ using biosignal amplifiers (g.USBamp) with filters between 0.1 and $100 \mathrm{~Hz}$ for EEG, 0.1 and $500 \mathrm{~Hz}$ for EMG, and a notch filter $(50 \mathrm{~Hz})$. Fig. 1 illustrates the paradigm timing, the cues on screen, the online feedback, and the EEG montage.

Cortical activation: Laplacian filters were applied to the EEG data to improve the SNR [7]. Afterwards, the EEG was filtered between 6 and $40 \mathrm{~Hz}$ and downsampled to $600 \mathrm{~Hz}$. Incorrectly performed trials (wrong leg movement, left/right co-contraction, late reaction time) and trials with artifacts $(\mathrm{EEG}>25 \mu \mathrm{V})$ were rejected. Only nine derivations close to electrode position $\mathrm{Cz}$ were considered (see Fig. 1), because ERD and ERS related to foot movements are prominent at the vertex $[8,9]$. Onset and offset of in- 
dividual movements were identified from the TA signals and used as triggers. The analysis focused on the perimovement ERD and the post-movement ERS. Frequency bands showing the strongest ERD or ERS (average from $500 \mathrm{~ms}$ to $1.5 \mathrm{~s}$ relative to movement onset/offset) were selected for further analysis. ERD and ERS waveforms were computed for each patient using a participant-specific frequency band. To summarize cortical activation, ERD was integrated in a $2.5 \mathrm{~s}$ window following movement onset, whereas ERS was integrated between $500 \mathrm{~ms}$ and $1.5 \mathrm{~s}$, relative to movement offset.

Statistical analysis: Statistical significance was evaluated for the ERD and ERS independently, with two-way ANOVAs with factors SIDE $\times$ ELECTRODE. The factor SIDE had two levels: affected vs. unaffected side of the body, whereas the factor ELECTRODE had nine levels: anterior-ipsilateral, anterior-central, anterior-contralateral, central-ipsilateral, vertex, central-contralateral, posterioripsilateral, posterior-central, and posterior-contralateral; relative to the affected side of the body. Significance values were corrected (Greenhouse-Geiser) if sphericity violations occurred.

\section{Results}

Peri-movement ERD: The average frequency band for ERD was $10.7 \pm 5.2$ and $22.4 \pm 7.5 \mathrm{~Hz}$. Significant differences were found for the main factor $\operatorname{SIDE}(\mathrm{F}(1,10)=$ $8.21, \mathrm{p}<0.05)$, indicating that the ERD was stronger during movements of the affected foot $(-381 \pm 293)$ than during movements of the unaffected foot $(-275 \pm 226)$.

Post-movement ERS: The average frequency band for ERS was $17.1 \pm 5.1$ and $24.1 \pm 6.1 \mathrm{~Hz}$. Significant differences were found for the main factor ELECTRODE $(\mathrm{F}(8,80)=4.11, \mathrm{p}<0.05$, Greenhouse-Geiser corrected $)$ and for the interaction SIDE $\times$ ELECTRODE $(F(8,80)=2.34$, $\mathrm{p}<0.05)$. Multiple comparisons between electrode positions revealed significant differences $(\mathrm{p}<0.05$, Bonferroni corrected) between the vertex and the electrode positions anterior ipsilateral, anterior contralateral, and central contralateral, i.e., the ERS at the vertex is stronger (147 \pm 171$)$ than the anterior locations (ipsilateral: $9 \pm 66$, contralateral: $45 \pm 90$ ) and the central contralateral to the affected foot (affected hemisphere: $10 \pm 75$ ). The multiple comparisons did not show significant differences for the interaction SIDE $\times$ ELECTRODE.

\section{Discussion}

The aim of our work was to investigate the cortical activation patterns of foot movements in subacute stroke patients. We found higher levels of cortical activity (ERD) for movements of the affected foot than during movements of the unaffected foot. Also, the post-movement response (ERS) at the vertex was stronger than pre-central positions and the central-contralateral to the affected foot (i.e., affected hemisphere). Experimental sessions of cue-paced active foot movement (with feedback) could be used as a novel strategy for motor rehabilitation. Online monitoring of cortical activation and comparisons between movements of both feet, could be useful to assess motor recovery (i.e., changes in cortical activation) $[4,5,6]$. Further work will focus on the post-movement ERS and possible differences between subacute stroke patients and healthy persons.

\section{Acknowledgement}

This work was supported by the FP7 EU Research Project BETTER (247935). The authors are thankful to Silvia Mena del Horno and Javier Bonilla for assistance during the experiments.

\section{Bibliography}

[1] J. J. Daly and J. R. Wolpaw, "Brain-computer interfaces in neurological rehabilitation," The Lancet Neurology, vol. 7, pp. 1032-1043, 2008. Review of BCIs in neurological rehabilitation.

[2] M. A. Dimyan and L. G. Cohen, "Neuroplasticity in the context of motor rehabilitation after stroke.," Nature Reviews Neurology, vol. 7, pp. 76-85, 2011.

[3] G. Pfurtscheller and F. H. Lopes da Silva, "Eventrelated EEG/MEG synchronization and desynchronization: basic principles," Clinical Neurophysiology, vol. 110, pp. 1842-1857, 1999.

[4] M. Stępień, J. Conradi, G. Waterstraat, F. U. Hohlefeld, G. Curio, and V. V. Nikulin, "Event-related desynchronization of sensorimotor EEG rhythms in hemiparetic patients with acute stroke.," Neuroscience Letters, vol. 488, pp. 17-21, Jan 2011.

[5] V. Kaiser, I. Daly, F. Pichiorri, D. Mattia, G. R. MüllerPutz, and C. Neuper, "Relationship between electrical brain responses to motor imagery and motor impairment in stroke," Stroke, 2012.

[6] K. Laaksonen, E. Kirveskari, J. P. Mäkelä, M. Kaste, S. Mustanoja, L. Nummenmaa, T. Tatlisumak, and N. Forss, "Effect of afferent input on motor cortex excitability during stroke recovery.," Clinical Neurophysiology, vol. 123, pp. 2429-2436, Dec 2012.

[7] B. Hjorth, "An on-line transformation of EEG scalp potentials into orthogonal source derivations," Electroencephalography and Clinical Neurophysiology, vol. 39, pp. 526-530, 1975.

[8] C. Neuper and G. Pfurtscheller, "Post movement synchronization of beta rhythms in the EEG over the cortical foot area in man," Neuroscience Letters, vol. 216, pp. 17-20, 1996.

[9] G. R. Müller-Putz, D. Zimmermann, B. Graimann, K. Nestinger, G. Korisek, and G. Pfurtscheller, "Eventrelated beta EEG-changes during passive and attempted foot movements in paraplegic patients," Brain Research, vol. 1137, pp. 84-91, 2007. 